\title{
Micromorfologia da superfície do fruto de espécies de Mikania Willd. (Asteraceae) ocorrentes no Estado do Rio Grande do Sul, Brasil ${ }^{1}$
}

\author{
Mara Rejane Ritter ${ }^{2,3}$ e Silvia Teresinha Sfoggia Miotto ${ }^{2}$
}

Recebido em 15/12/2004. Aceito em 16/08/2005.

\begin{abstract}
RESUMO - (Micromorfologia da superfície do fruto de espécies de Mikania Willd. (Asteraceae) ocorrentes no Estado do Rio Grande do Sul, Brasil). A micromorfologia da superfície dos frutos de 15 táxons de Mikania Willd. em microscopia eletrônica de varredura (MEV) é apresentada neste trabalho. Buscaram-se caracteres que auxiliassem a separação de espécies próximas, ocorrentes no Estado do Rio Grande do Sul. Para algumas delas, estes caracteres mostraram valor taxonômico. As espécies estudadas foram divididas em quatro grupos, de acordo com o padrão encontrado. O padrão que apresentou paredes periclinais estriadas e paredes anticlinais com projeções verrucosas foi o mais constante nas espécies de Mikania Willd. analisadas.
\end{abstract}

Palavras-chave: Mikania, Asteraceae, cipsela, microscopia eletrônica de varredura

\begin{abstract}
Micromorphology of fruit surfaces in species of Mikania Willd. (Asteraceae) occurring in Rio Grande do Sul State, Brazil). This work presents the surface micromorphology of fruits of 15 taxa of Mikania Willd. studied under scanning electron microscopy (SEM). The aim was to find characters to separate close species that occur in Rio Grande do Sul State. Such characters had taxonomic value for some of them. The species were separated into four groups according to the pattern. The pattern with striated periclinal and anticlinal walls with verrucose projections was the most constant in the species of Mikania Willd. analyzed.
\end{abstract}

Key words: Mikania, Asteraceae, cypsela, scanning electron microscopy

\section{Introdução}

Mikania Willd., da tribo Eupatorieae, é um gênero principalmente neotropical, com algumas espécies distribuídas na América temperada e nos paleotrópicos (Cabrera et al. 1996).

Este gênero é considerado taxonomicamente estável, por apresentar caracteres morfológicos florais constantes, embora as espécies sejam de difícil delimitação. Robinson (1934) mencionou a ocorrência de "complexos" em Mikania Willd. A identificação das espécies por meio de caracteres morfológicos tradicionais vem sendo complementada pela busca de novos caracteres diagnósticos para a separação dos táxons.

Em diferentes grupos vegetais, uma das abordagens que tem apresentado resultados satisfatórios é a da análise da micromorfologia da superfície de sementes e de frutos em microscopia eletrônica de varredura. Sementes e pequenos frutos apresentam uma complexa diversidade morfológica e micromorfológica, com informações de valor taxonômico. $\mathrm{O}$ exame da superfície epidérmica por meio do microscópio eletrônico de varredura (MEV) tem revelado caracteres diagnósticos importantes. Segundo Silveira (1998), o MEV forma imagens tridimensionais e de grande efeito plástico, com uma notável profundidade de foco. Segundo Barthlott (1984; 1990), esses caracteres são surpreendentemente pouco afetados por condições ambientais, sendo constantes e confiáveis para espécies ou grupos de espécies.

Estudos da superfície de sementes e de frutos foram realizados em diferentes grupos, como os de Walter (1975) em Carex L. (Cyperaceae), Carolin (1980) em Goodenia Sm. (Goodeniaceae), Chuang \& Heckard (1983) em Orthocarpus Nutt (Scrophulariaceae), Denton (1983) em Cyperus L. (Cyperaceae), Edmonds (1983) em Solanum L. (Solanaceae), Farooqui \& Bahadur (1985; 1986) em Nicotiana L. (Solanaceae), Chuang \& Ornduff (1992)

\footnotetext{
1 Parte da Tese de Doutorado do primeiro Autor

2 Universidade Federal do Rio Grande do Sul, Departamento de Botânica, Instituto de Biociências, Av. Bento Gonçalves, 9500, Setor IV, CEP 91509-900, Campus do Vale, Porto Alegre, RS, Brasil

3 Autor para correspondência: mrritter@terra.com.br
} 
em Menianthaceae, Araújo \& Longhi-Wagner (1997) em Cyperus L. (Cyperaceae), Boechat \& LonghiWagner (2003) em Eragrostis Wolf (Poaceae), Suseela et al. (1998) em Nymphoides hydrophylla (Lour.) Kuntze (Nympheaceae), Carvalho et al. (1999) em Schwenckia L. (Solanaceae) e Zanin \& LonghiWagner (2001) em Andropogon L. (Poaceae).

Alguns autores utilizaram o MEV para analisar a superfície de frutos em Asteraceae, destacando-se os trabalhos de Anderberg (1991) para a tribo Gnaphalieae, Kim \& Turner (1992) em Krigia Schreb. (Lactuceae), Cron et al. (1993) em Cineraria L. (Senecioneae), Freire \& Katinas (1995) em Nassauviinae (Mutisieae), Ouyahya (1995) em Artemisia L. (Anthemideae), Tadesse et al. (1995) em Coreopsis L. e Bidens L. (Heliantheae), Tadesse \& Reilly (1995) em Helichrysum Mill. (Gnaphalieae), Hook \& Reid (1996) em Leontopodium alpinum Cass. (Gnaphalieae), Sennikov \& Illarionova (1999) em Prenanthes pendula Sch. Bip. (Lactuceae), Zarembo \& Boyko(1999) em Atractylodes DC. (Cynareae), Smalla (2000) em Hypochaeridinae (Lactuceae) e Mukherjee \& Sarkar (2001) em Astereae.

Brisson \& Peterson (1976) afirmaram que espécies da família Asteraceae apresentam diferentes tipos de tricomas e uma redução nas ornamentações das paredes celulares das células epidérmicas do pericarpo.

O objetivo deste trabalho é verificar se as características da superfície dos frutos em Microscopia Eletrônica de Varredura auxiliariam na identificação de espécies próximas do gênero Mikania Willd.

\section{Material e métodos}

Na preparação das amostras, sempre que possível, procurou-se selecionar, no mínimo, três exemplares férteis de cada espécie avaliada. Preparou-se sempre de duas a três cipselas maduras, sem resíduos e sem fungos. Os frutos foram montados diretamente em suportes porta-amostras para MEV (stubs), sem tratamento prévio, fixados com esmalte incolor. As amostras foram metalizadas com ouro com $20 \mathrm{~nm}$ de espessura, por aproximadamente 80 segundos, em corrente de $40 \mathrm{~mA}$, em metalizador BAL-TEC $®$ modelo SCD 050. Em seguida, foram analisadas em microscópio eletrônico de varredura JEOL $®$ JSM-5800 Scanning Microscope, no Centro de Microscopia Eletrônica (CME) da Universidade Federal do Rio Grande do Sul. As imagens obtidas foram gravadas em disquete e, posteriormente, selecionadas e referem-se a frutos que sofreram dessecação em estufa ou em processo natural, podendo ocorrer artefatos que modificam a sua interpretação. A terminologia utilizada neste trabalho baseia-se em Barthlott (1984; 1990).

Os exemplares selecionados para este estudo estão listados abaixo.

Material examinado: Mikania capricorni B.L. Rob.: M.R. Ritter 630 (ICN), J. Mattos \& N. Mattos 23166 (HAS), G. Hatschbach 21162 (PEL); Mikania clematidifolia Dusén: Cordeiro \& E. Barbosa 811 (C); Mikania cordifolia (L.f.) Willd.: M.R. Ritter 1098 (ICN), M.R. Ritter 1206 (ICN), M.R. Ritter 1250 (ICN), M.R. Ritter 1311 (ICN), M.R. Ritter 1355 (ICN), M.R. Ritter 1371 (ICN); Mikania cynanchifolia Hook. \& Arn. ex B.L. Rob.: K. Hagelund 2242 (ICN), Albuquerque s.n. (ICN 87564), Nhuch s.n. (ICN 69761); Mikania dusenii B.L. Rob.: M.N. Correa 5329 (BAB); Mikania glomerata Spreng.: C. Jurinitz s.n. (ICN 119090), M.R. Ritter 1392 (ICN), S.L. Carvalho Leite s.n. (ICN 84431); Mikania laevigata Sch. Bip. ex Baker: M.R. Ritter 701 (ICN), S. Marodin 209 (ICN), S.L. Carvalho Leite s.n. (ICN 120519); Mikania micrantha Kunth: M.R. Ritter 1112 (ICN), M.R. Ritter 1119 (ICN), M.R. Ritter 1139 (ICN); Mikania oreophila M.R. Ritter \& Miotto: M.R. Ritter 1162 (ICN), N. Silveira \& C. Mansan 11924 (ICN), R.J.F. Garcia et al. 772 (PMSP); Mikania paranensis Dusén: M.R. Ritter 1003 (ICN), M.R. Ritter 1151 (ICN), M.R. Ritter 1160 (ICN); Mikania parodii Cabrera: M.R. Ritter 836 (ICN), M.L. Abruzzi 770 (ICN), L. Roth 89 (ICN); Mikania periplocifolia Hook. \& Arn.: M.R. Ritter \& F.A. Silva F. 116 (ICN), M.R. Ritter 1093 (ICN), Rodrigues 943 (ICN); Mikania ternata (Vell.) B.L. Rob.: M.R. Ritter 712 (ICN), M. Sobral 2059a (ICN), K. Hagelund 15216 (ICN), Silva et al. 589 (PEL), L.C.Q.M.P. Sampaio \& R.J.F. Garcia 241 (SPF), Fernandes \& Santos 70 (UPCB); Mikania trachypleura B.L. Rob.: G. Hatschbach 36612 (C, Z); Mikania ulei Hieron.: M.R. Ritter 1389 (ICN), Wongtschowski et al. 29 (SPF), Gaelzer 295 (ICN).

\section{Resultados e discussão}

As cipselas de Mikania Willd. são glabras ou pilosas, 5-costadas e, mais raramente, 10 -costadas. São normalmente enegrecidas quando maduras, pela presença de fitomelanina no fruto, utilizando-se o termo cipselas carbonizadas. Segundo Robinson (1996), esta característica ocorrente nas espécies de Heliantheae 
e Eupatorieae evidencia a relação entre as duas tribos. No ápice das cipselas encontra-se o papus formado por numerosas cerdas que têm um papel muito importante na dispersão dos frutos.

A superfície das cipselas em algumas espécies de Mikania Willd., observada em estereomicroscópio, mostrava-se predominantemente estriada. Com a análise em microscopia eletrônica de varredura, outros padrões foram verificados (Tab. 1).

Em relação à escultura primária, detectou-se a ocorrência de projeções verrucosas na parede anticlinal longitudinal, além de paredes anticlinais longitudinais lisas. Quanto à escultura secundária, a maioria das espécies analisadas apresentou o padrão de ornamentação estriado ou liso.

As estrias medem cerca de $10 \mu \mathrm{m}$ de largura em Mikania paranensis Dusén, enquanto que nas outras espécies, as estrias medem cerca de $2 \mu \mathrm{m}$ de largura. Estas estrias muitas vezes formam bandas, que medem cerca de 10-15 $\mu \mathrm{m}$ de largura como em Mikania glomerata Spreng.

As espécies analisadas foram agrupadas de acordo com as afinidades morfológicas e taxonômicas entre elas.
1. Mikania glomerata Spreng. e Mikania laevigata Sch. Bip. ex Baker

Em Mikania glomerata Spreng. ocorrem cerca de quatro estrias por banda, além de alguns tricomas simples, pluricelulares, unisseriados na superfície dos frutos.

Em Mikania laevigata Sch. Bip. ex Baker ocorrem de três a quatro estrias por banda, algumas vezes com bandas de difícil delimitação. Também ocorrem alguns tricomas simples, pluricelulares, unisseriados e glandulares na superfície dos frutos.

O padrão encontrado nestas duas espécies é semelhante ao encontrado por Cron et al. (1993) em Cineraria atriplicifolia DC. (Senecioneae), que apresentou bandas com cerca de dez estrias. Outros autores encontraram este padrão de estrias longitudinais na superfície das cipselas, como Ouyahya (1995) em Artemisia verlotorum Lamotte (Anthemideae) e Tadesse et al. (1995) na maioria das espécies de Bidens L. (Heliantheae). Mukherjee \& Sarkar (2001) encontraram a superfície fracamente estriada em Myriactis humilis Merrill (Astereae). O padrão estriado foi encontrado em algumas espécies

Tabela 1. Características da superfície de frutos de algumas espécies de Mikania ocorrentes no Estado do Rio Grande do Sul, observadas em Microscopia Eletrônica de Varredura

\begin{tabular}{|c|c|c|c|c|c|}
\hline Espécies & $\begin{array}{c}\text { Projeções } \\
\text { verrucosas na } \\
\text { parede anticlinal } \\
\text { longitudinal }\end{array}$ & $\begin{array}{l}\text { Parede estrial } \\
\text { periclinal } \\
\text { externa bc } \\
\\
\end{array}$ & $\begin{array}{l}\text { Número de } \\
\text { estrias por } \\
\text { anda na parede } \\
\text { ericlinal externa }\end{array}$ & Tricomas & $\begin{array}{l}\text { Costelas } \\
\text { escabras }\end{array}$ \\
\hline \multicolumn{6}{|l|}{ Grupo 1} \\
\hline Mikania capricorni B.L. Rob. & ausentes & estriada & $5-7$ & simples & ausentes \\
\hline M. clematidifolia Dusén & ausentes & estriada & $4-5$ & glandulares & ausentes \\
\hline M. cordifolia (L.F.) Willd. & ausentes & estriada & 5 & simples e glandulares & presentes \\
\hline M. glomerata Spreng. & ausentes & estriada & 4 & simples & ausentes \\
\hline M. laevigata Sch. Bip. ex Baker & ausentes & estriada & $3-4$ & simples e glandulares & ausentes \\
\hline M. oreophila M.R. Ritter \& Miotto & ausentes & estriado-ondulada & $3-4$ & simples & ausentes \\
\hline M. paranensis Dusén & ausentes & estriada & $3-4$ & glandulares & ausentes \\
\hline \multicolumn{6}{|l|}{ Grupo 2} \\
\hline M. dusenii B.L. Rob. & ausentes & lisa & 0 & glandulares & ausentes \\
\hline \multicolumn{6}{|l|}{ Grupo 3} \\
\hline $\begin{array}{l}\text { M. cynanchifolia Hook. \& Arn. ex } \\
\text { B.L. Rob. }\end{array}$ & presentes & estriada & indefinidas & glandulares & ausentes \\
\hline M. micrantha Kunth & presentes & estriada & indefinidas & simples e glandulares & presentes \\
\hline M. periplocifolia Hook. \& Arn. & presentes & estriada & indefinidas & glandulares & ausentes \\
\hline M. ternata (Vell.) B.L. Rob. & presentes & estriada & 7 & simples & ausentes \\
\hline M. trachypleura B.L. Rob. & presentes & estriada & indefinidas & simples e glandulares & presentes \\
\hline \multicolumn{6}{|l|}{ Grupo 4} \\
\hline M. parodii Cabrera & presentes & lisa & 0 & glandulares & ausentes \\
\hline M. ulei Hieron. & presentes & lisa & indefinidas & simples e glandulares & presentes \\
\hline
\end{tabular}


de Eragrostis Wolf (Poaceae) por Boechat \& LonghiWagner (2003).

O padrão estriado encontrado em Mikania glomerata Spreng. e Mikania laevigata Sch. Bip. ex Baker não permite estabelecer diferenças seguras que diferenciem estas duas espécies por características da superfície das cipselas.

\section{Mikania oreophila M.R. Ritter \& Miotto e Mikania paranensis Dusén}

Em Mikania oreophila M.R. Ritter \& Miotto ocorrem cerca de três a quatro estrias onduladas por banda, algumas vezes com bandas de difícil delimitação (Fig. 1A). Esta ondulação observada pode ter ocorrido devido ao processo de secagem da planta. São encontrados alguns tricomas simples, pluricelulares, unisseriados na superfície dos frutos. O padrão ondulado foi encontrado em Krigia Schreb. (Lactuceae) por Kim \& Turner (1992) e em algumas espécies de Andropogon L. (Poaceae) por Zanin \& Longhi-Wagner (2001).

Em Mikania paranensis Dusén ocorrem cerca de três a quatro estrias levemente onduladas por banda, algumas vezes com bandas de difícil delimitação. São encontrados muitos tricomas glandulares na superfície dos frutos desta espécie.

A presença de tricomas glandulares foi constatada por Mukherjee \& Sarkar (1997) em algumas espécies de Eupatorieae. Em Mikania trinervis Hook. \& Arn. foram encontrados tricomas glandulares unisseriados, não ramificados, multicelulares, com células glandulares terminais e vesículas subglobosas subsésseis.

Mikania oreophila M.R. Ritter \& Miotto e Mikania paranensis Dusén distinguem-se pela ocorrência de tricomas simples e glandulares, respectivamente. $\mathrm{O}$ padrão da superfície não permite estabelecer diferenças seguras que permitam o reconhecimento das espécies, já que nas duas ocorre o padrão estriado.

\section{Mikania clematidifolia Dusén, Mikania ternata} (Vell.) B.L. Rob. e Mikania ulei Hieron.

Em Mikania clematidifolia Dusén ocorrem cerca de quatro a cinco estrias por banda, algumas vezes com bandas de difícil delimitação. Tricomas glandulares, pluricelulares, unisseriados, com células glandulares terminais, esparsos, também são encontrados na superfície dos frutos (Fig. 1B).

Em Mikania ternata (Vell.) B.L. Rob. são encontradas cerca de sete estrias onduladas por banda, com bandas de difícil delimitação. Na parede anticlinal longitudinal ocorrem projeções verrucosas (Fig. 1C).

Em Mikania ulei Hieron. ocorrem células poligonais com a superfície lisa. Em algumas regiões do fruto ocorrem projeções verrucosas nas paredes anticlinais longitudinais (Fig. 1D). Na superfície dos frutos ocorrem alguns tricomas simples, pluricelulares, unisseriados e alguns glandulares. Verificou-se ainda a presença de costelas escabras.

Projeções verrucosas foram encontradas por Tadesse et al. (1995) em várias espécies de Bidens L. (Heliantheae), bem como em Eragrostis Wolf (Poaceae) por Boechat \& Longhi-Wagner (2003) e em Andropogon L. (Poaceae) por Zanin \& LonghiWagner (2001).

Analisando-se o padrão de superfície destas três espécies, é possível distinguí-las. Mikania clematidifolia Dusén e Mikania ternata (Vell.) B.L. Rob. são semelhantes, mas a parede anticlinal com projeções verrucosas em Mikania ternata (Vell.) B.L. Rob. é marcante. Mikania ulei Hieron. é seguramente distinta, com a ocorrência de células poligonais, padrão não encontrado nas outras duas espécies.

4. Mikania cordifolia (L.f.) Willd., Mikania cynanchifolia Hook. \& Arn. ex B.L. Rob., Mikania micrantha Kunth e Mikania trachypleura B.L. Rob.

Em Mikania cordifolia (L.f.) Willd. o padrão apresentado é o de cerca de cinco estrias por banda. Alguns tricomas simples, pluricelulares, unisseriados e glandulares também ocorrem na superfície dos frutos. As costelas são escabras.

Foram encontradas em Mikania cynanchifolia Hook. \& Arn. ex B.L. Rob. projeções verrucosas na parede anticlinal longitudinal e estrias na parede periclinal, além de tricomas glandulares esparsos na superfície dos frutos.

Em Mikania micrantha Kunth ocorrem projeções verrucosas na parede anticlinal longitudinal e estrias na parede periclinal. Foram encontrados alguns tricomas simples, pluricelulares, unisseriados e muitos tricomas glandulares na superfície dos frutos. As costelas são escabras (Fig. 1E).

Em Mikania trachypleura B.L. Rob. ocorrem projeções verrucosas na parede anticlinal longitudinal, estrias na parede periclinal e tricomas simples, pluricelulares, unisseriados, além de tricomas glandulares na superfície dos frutos. As costelas são escabras.

Das quatro espécies analisadas, Mikania cynanchifolia Hook. \& Arn. ex B.L. Rob. distinguese das três restantes por não possuir costelas escabras. 

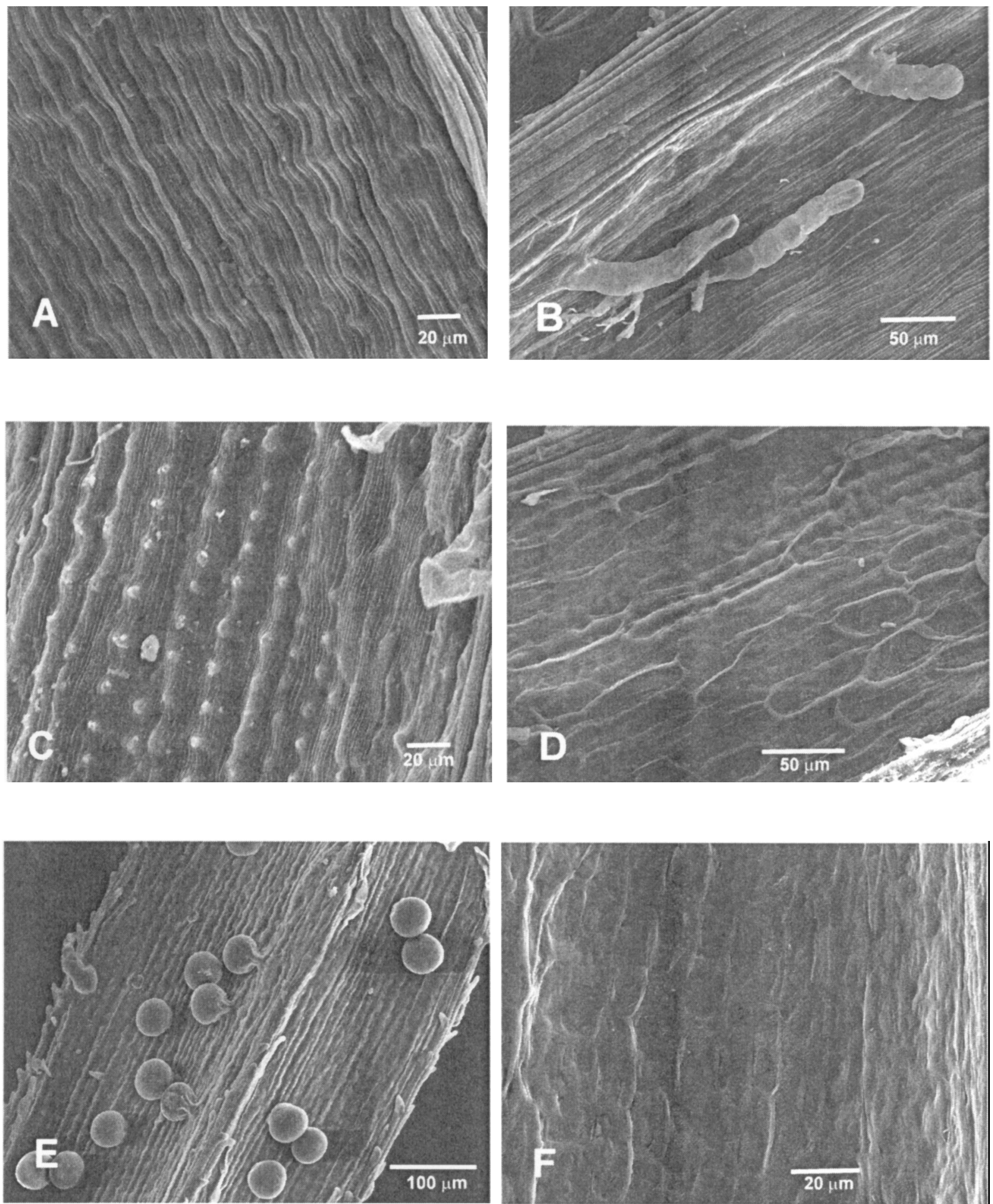

Figura 1. Detalhes da superfície de frutos em MEV - A. Mikania oreophila M.R. Ritter \& Miotto - grupo 1 (Silveira \& Mansan 11924, HAS). B. Mikania clematidifolia Dusén - grupo 1 (Cordeiro \& Barbosa 811, C). C. Mikania ternata (Vell.) B.L. Rob. - grupo 3 (Sampaio \& Garcia 241, SPF). D. Mikania ulei Hieron. - grupo 4 (Wongtschowsky et al. 29, SP). E. Mikania. micrantha Kunth - grupo 3 (Ritter 1139, ICN). F. Mikania dusenii B.L. Rob. - grupo 2 (Correa 5329, BAB). 
Mikania cordifolia (L.f.) Willd. distingue-se das demais por não apresentar projeções verrucosas. Já em Mikania micrantha Kunth e Mikania trachypleura B.L. Rob. o padrão da superfície não permite estabelecer diferenças seguras que permitam o reconhecimento das duas espécies, já que em ambas ocorre o mesmo padrão.

5. Mikania capricorni B.L. Rob., Mikania dusenii B.L. Rob., Mikania parodii Cabrera e Mikania periplocifolia Hook. \& Arn.

Em Mikania capricorni B.L. Rob. ocorre o padrão estriado na parede periclinal. Ocorrem tricomas simples, pluricelulares, unisseriados esparsos sobre a superfície dos frutos.

Células poligonais com parede periclinal lisa (Fig. 1F) e, tricomas glandulares na superfície dos frutos, são encontrados em Mikania dusenii B.L. Rob.

Em Mikania parodii Cabrera ocorrem células poligonais com parede periclinal lisa, além de projeções verrucosas nas paredes anticlinais longitudinais. Tricomas glandulares são encontrados na superfície dos frutos.

Já Mikania periplocifolia Hook. \& Arn. apresenta projeções verrucosas nas paredes anticlinais longitudinais e estrias na parede periclinal. São encontrados tricomas glandulares na superfície dos frutos.

O padrão de paredes lisas foi encontrado também por Anderberg (1991) na tribo Gnaphalieae. Células poligonais foram encontradas por Zarembo \& Boyko (1999) em Atractylodes ovata (Thunb.) DC. (Cynareae). Segundo Mukherjee \& Sarkar (1997) Eupatorium cannabinum L. possui superfície lisa. Este padrão também foi encontrado por Araujo \& Longhi-Wagner (1997) em Cyperus L. (Cyperaceae), por Boechat \& Longhi-Wagner (2003) em Eragrostis Wolf (Poaceae) e por Zanin \& Longhi-Wagner (2001) em Andropogon L. (Poaceae).

Das quatro espécies analisadas, Mikania capricorni B.L. Rob. distingue-se das demais por não possuir células poligonais e projeções verrucosas. Das três restantes, Mikania periplocifolia Hook. \& Arn. diferencia-se por não apresentar células poligonais. Em Mikania parodii Cabrera ocorre o padrão com projeções verrucosas, diferentemente de Mikania dusenii B.L. Rob.

O estudo dos caracteres micromorfológicos da superfície dos frutos em Microscopia Eletrônica de Varredura em Mikania Willd., mostrou-se eficiente para a separação de algumas espécies próximas. Para outras, estes padrões são semelhantes e não apresentaram caráter diagnóstico. Diferenças morfológicas puderam ser observadas em alguns grupos de espécies, como por exemplo, em Mikania oreophila M.R. Ritter \& Miotto e Mikania paranensis Dusén, em Mikania clematidifolia Dusén, Mikania ternata (Vell.) B.L. Rob. e Mikania ulei Hieron., em Mikania cynanchifolia Hook. \& Arn. ex B.L. Rob. e Mikania cordifolia (L.f.) Willd. e por fim, em Mikania capricorni B.L. Rob., Mikania dusenii B.L. Rob., Mikania parodii Cabrera e Mikania periplocifolia Hook. \& Arn.

Com base nos caracteres morfológicos verificados nos 15 táxons estudados, e independentemente da sua proximidade taxonômica, foi possível classificá-los em quatro grupos:

1) Grupo 1: espécies com parede periclinal estriada e estrias organizadas em bandas: Mikania capricorni B.L. Rob., Mikania clematidifolia Dusén, Mikania cordifolia (L.f.) Willd., Mikania glomerata Spreng., Mikania laevigata Sch. Bip. ex Baker, Mikania oreophila M.R. Ritter \& Miotto e Mikania paranensis Dusén.

2) Grupo 2: espécie com parede periclinal lisa, delimitada por células poligonais: Mikania dusenii B.L. Rob.

3) Grupo 3: espécies com parede anticlinal longitudinal com projeções verrucosas: Mikania cynanchifolia Hook. \& Arn. ex B.L. Rob., Mikania micrantha Kunth, Mikania periplocifolia Hook. \& Arn., Mikania ternata (Vell.) B.L. Rob. e Mikania trachypleura B.L. Rob.

4) Grupo 4: espécies com parede anticlinal longitudinal com projeções verrucosas e parede periclinal lisa delimitada por células poligonais: Mikania parodii Cabrera e Mikania ulei Hieron.

Os padrões de superfície de fruto encontrados mostraram-se parcialmente eficazes na resolução de problemas taxonômicos de algumas espécies próximas de Mikania Willd..

\section{Referências bibliográficas}

Anderberg, A.A. 1991. Taxonomy and phylogeny of the tribe Gnaphalieae (Asteraceae). Opera Botanica 104: 1-195.

Araújo, A.C. \& Longhi-Wagner, H.M. 1997. Anatomia foliar e micromorfologia do fruto na taxonomia de Cyperus L. (Cyperaceae). Iheringia, série Botânica 48: 103-104.

Barthlott, W. 1984. Microstructural features of seed surfaces. Pp. 95-105. In: V.H. Heywood \& D.M. Moore (eds.). Current concepts in plant taxonomy. London, Academic Press. 
Barthlott, W. 1990. Scanning electron microscopy of the epidermal surface in plants. Pp. 69-94. In: D. Claugher (ed.). Scanning Electron Microscopy in Taxonomy and Functional Morphology. Oxford, Clarendon Press.

Boechat, S.C. \& Longhi-Wagner, H.M. 2003. Análise do fruto em espécies de Eragrostis Wolf (Poaceae). Iheringia, série Botânica 58(1): 1-168.

Brisson, J.D. \& Peterson, R.L. 1976. A critical review of the use of scanning electron microscopy in the study of the seed coat. Scanning Electron Microscopy 7: 477-496.

Cabrera, A.L.; Holmes, W.C. \& McDaniel, S. 1996. Compositae III, Asteroideae, Eupatorieae. Flora del Paraguay 25: 208-273.

Carolin, R.C. 1980. Pattern of the seed surface of Goodenia and related genera. Australian Journal Botany 28: 123-137.

Carvalho, L.d'A.F.; Machado, R.D. \& Bovini, M.G. 1999. Seed coat micromorphology of brasilian species of Schwenckia. Pp. 22-32. In: M. Nee; D.E. Symon; R.N. Lester et al. (eds.). Solanaceae IV. Kew, Royal Botanic Gardens.

Chuang, T.I. \& Heckard, L.R. 1983. Systematic significance of seed-surface features in Orthocarpus (Scrophulariaceae-subtribe Castillejinae). American Journal of Botany 70(6): 877-890.

Chuang, T.I. \& Ornduff, R. 1992. Seed morphology and systematics of Menyanthaceae. American Journal of Botany 79(12): 1396-1406.

Cron, G.V.; Robbertse, P.J. \& Vincent, P.L.D. 1993. The anatomy of the cypselae of species of Cineraria L. (Asteraceae-Senecioneae) and its taxonomic significance. Botanical Journal of the Linnean Society 112: 319-334.

Denton, M.F. 1983. Anatomical studies of the "Luzulae" group of Cyperus (Cyperaceae). Systematic Botany 8(3): 250-262.

Edmonds, J.M. 1983. Seed coat structure and development in Solanum L. section Solanum (Solanaceae). Botanical Journal of the Linnean Society 87: 229-246.

Farooqui, S.M. \& Bahadur, B. 1985. Studies on seed morphology (LM \& SEM) of american Nicotiana L. (Solanaceae). Indian Journal Botany 8(2): 191-197.

Farooqui, S.M. \& Bahadur, B. 1986. Seed and seed coat characters in australian Nicotiana. Pp. 114-137. In: W.G. D'Arcy (ed.). Solanaceae-Biology and Systematics. Columbia Univerity Press.

Freire, S.E. \& Katinas, L. 1995. Morphology and ontogeny of the cypsela hairs of Nassauviinae (Asteraceae, Mutisieae). Pp. 107-143. In: D.J.N. Hind; C. Jeffrey \& G.V. Pope (eds.). Advances in Compositae Systematics. Kew, Royal Botanicals Gardens.

Hook, I. \& Reid, C. 1996. The microscopic structure of Leontopodium alpinum (Edelweiss). Pp. 383-392. In: P.D.S. Caligari \& D.J.N. Hind (eds.). Compositae: Biology and Utilization. v.2. Kew, Royal Botanicals Gardens.
Kim, K. \& Turner, B.L. 1992. Systematic overview of Krigia (Asteraceae-Lactuceae). Brittonia 44(2): 173-198.

Mukherjee, S.K. \& Sarkar, A. 1997. Morphological and anatomical structure of the cypselas of some species of the tribe Eupatorieae (Asteraceae). Journal Plant Anatomy and Morphology 7(1): 9-19.

Mukherjee, S.K. \& Sarkar, A. 2001. Morphology and structure of cypselas in thirteen species of the tribe Astereae (Asteraceae). Phytomorphology 51(1): 17-26.

Ouyahya, A. 1995. Systematique du genre Artemisia au Maroc. Pp. 293-354. In: D.J.N. Hind; C. Jeffrey \& G.V. Pope (eds.). Advances in Compositae Systematics. Kew, Royal Botanicals Gardens.

Robinson, B.L. 1934. Mikania scandens and its near relatives. Contribuitions Gray Herbarium 104: 55-71.

Robinson, H. 1996. Recent studies in the Heliantheae and Eupatorieae. Pp. 627-653. In: P.D.S. Caligari \& D.J.N. Hind (eds.). Compositae: Systematics. v.1. Kew, Royal Botanicals Gardens.

Sennikov, A.N. \& Illarionova, I.D. 1999. Reclassification of Prenanthes pendula (Asteraceae: Lactuceae). Compositae Newsletter 34: 53-61.

Silveira, M. 1998. Preparo de amostras biológicas para microscopia eletrônica de varredura. Pp. 33-37. In: W. Souza (ed.). Técnicas básicas de microscopia eletrônica aplicadas às Ciências Biológicas. Rio de Janeiro, Sociedade Brasileira de Microscopia.

Smalla, M. 2000. Studies in the Compositae of the Arabian Peninsula and Socotra - 6. The Hypochaeridinae (Lactuceae) in the Arabian Peninsula. Willdenowia 30(2): 315-337.

Suseela, M.R.; Rai, U.N.; Gupta, P. \& Devi, S. 1998. Cell wall ultrastructure of the testa of Nymphoides hidrophylla (Lour.) Kuntze. Phytomorphology 48(3): 289-293.

Tadesse, M. \& Reilly, T. 1995. A contribution to studies on Helichrysum (Compositae-Gnaphalieae) - a revision of the species of north-east tropical Africa. Pp. 379-450. In: D.J.N. Hind; C. Jeffrey \& G.V. Pope (eds.). Advances in Compositae Systematics. Kew, Royal Botanicals Gardens.

Tadesse, M.; Crawford, D.J. \& Smith, E.B. 1995. Comparative capitular morphology and anatomy of Coreopsis L. and Bidens L. (Compositae), including a review of generic boundaries. Brittonia 47(1): 61-91.

Walter, K.S. 1975. A preliminary study of the achene epidermis of certain Carex (Cyperaceae) using scanning electron microscopy. Michigan Botanist 14: 67-71.

Zanin, A. \& Longhi-Wagner, H.M. 2001. Micromorfologia da superfície de fruto em espécies de Andropogon L. (Poaceae-Andropogoneae) ocorrentes no Brasil. Insula 30: $35-46$.

Zarembo, E.V. \& Boyko, E.V. 1999. The genus Atractylodes DC. (Compositae-Cynareae) in Far East Russia. Compositae Newsletter 33: 61-72. 